\title{
A sensitivity approach to modeling longitudinal bivariate ordered data subject to informative dropouts
}

\author{
D. Todem $\cdot$ K. Kim $\cdot$ E. Lesaffre
}

Received: 19 November 2004/Revised: 23 March 2006/

Accepted: 30 May 2006/Published online: 4 October 2006

(C) Springer Science+Business Media, LLC 2006

\begin{abstract}
Incomplete data abound in epidemiological and clinical studies. When the missing data process is not properly investigated, inferences may be misleading. An increasing number of models that incorporate nonrandom incomplete data have become available. At the same time, however, serious doubts have arisen about the validity of these models, known to rely on strong and unverifiable assumptions. A common conclusion emerging from the current literature is the clear need for a sensitivity analysis. We propose in this paper a detailed sensitivity analysis using graphical and analytical techniques to understand the impact of missing-data assumptions on inferences. Specifically, we explore the influence of perturbing a missing at random model locally in the direction of non-random dropout models. Data from a psychiatric trial are used to illustrate the methodology.
\end{abstract}

Keywords Bivariate ordinal outcomes $\cdot$ Latent variable $\cdot$ Maximum marginal likelihood $\cdot$ Non-ignorable dropout $\cdot$ Non-parametric density estimation Repeated measures - Sensitivity analysis - Shared random effects · Threshold crossing model

\section{Introduction}

The problem of missing data and specifically that of dropouts is common throughout statistical work and is almost ever present in the analysis of longitudinal data. In

D. Todem $(\bowtie)$

Division of Biostatistics, Department of Epidemiology, Michigan State University, B601 West Fee Hall, East Lansing, MI 48823, USA

e-mail: todem@msu.edu

K. Kim

Department of Biostatistics \& Medical Informatics, University of Wisconsin-Madison, 600 Highland Ave., Madison, WI 53792, USA

E. Lesaffre

Biostatistical Centre, Katholieke Universiteit Leuven, Kapucijnenvoer 35, B-3000 Leuven, Belgium 
addition, subjects who drop out prematurely from a longitudinal study usually do not represent the ones who complete the study. For example, in psychiatric trials of a drug adverse response, subjects who terminate the study prematurely often exhibit more side effects relatively to their peers who complete the study. One is then interested in understanding the (potential) effects of incompleteness on statistical inferences. In a strict sense, the conventional justification of using randomization in many clinical trials could be affected when subjects drop out for reasons unknown to the investigator (Lesaffre et al. 1996).

The motivation for this study comes from a Fluvoxamine (a serotonin reuptake inhibitor) clinical trial. This is a non-comparative post-marketing study, designed to reflect clinical practice closely with out-patients diagnosed with depression, obsessive-compulsive disorder or panic disorder. Accumulated experience in controlled trials has shown that Fluvoxamine is as effective as conventional tricyclic antidepressant drugs, and more effective than placebo in the treatment of depression (for a review, see Burton 1991). However, many patients suffering from depression have concomitant morbidity associated with this condition. It was therefore decided to setup a post-marketing pharmaco-vigilance trial to study more accurately the profile of Fluvoxamine in ambulatory clinical psychiatric practice. A total of 315 patients with a diagnosis of either depression or obsessive-compulsive or panic disorder were enrolled in the study. All subjects were treated with Fluvoxamine in doses ranging from $100 \mathrm{mg} /$ day to $300 \mathrm{mg} / \mathrm{day}$ and underwent clinical evaluations at baseline, 2, 4, 8 and 12 weeks. The primary endpoints comprised the side effects of the drug and its therapeutic effect both recorded on an ordinal scale. The side effects were defined as adverse events occurring during the course of the trial but not present at baseline and were recorded as: (1) no side effects; (2) no significant interference with functionality of the patient; (3) significant interference with functionality of the patient; and (4) side effects surpass the therapeutic effects. The therapeutic response was defined as the effect of the medication on the symptoms occurring during the study and was recorded as: (1) no improvement or worsening; (2) minimal improvement; (3) moderate improvement; and (4) important improvement or complete disappearance of the symptoms. Several patient's baseline characteristics such as sex, age, initial severity of the disease on a 1-7 scale, and duration of the mental illness were recorded. At the end of the study, only 224 patients had a full-sequence data resulting from the fact that 14 subjects were not observed after recruitment, 31, 26, and 18 patients dropped out, respectively, after the first, second and third visit and two patients had a non-monotone missing pattern. The primary objective of this paper is to jointly study the time evolution of the side effects response as well as that of the therapeutic response, taking into account the dropout process.

Statistical analysis of longitudinal bivariate ordinal outcomes data raises a number of challenging issues. The clustering due to repeated observations from the same subject and the multiplicity of outcomes at each time point necessitate the use of methods for correlated data. In addition, the dropout process is another important issue. Using terminology from Little and Rubin (1987), missing data mechanisms are classified as missing completely at random (MCAR), missing at random (MAR) and missing not at random (MNAR), if missingness is allowed to depend (1) none of the outcomes, (2) the observed outcomes only, or (3) unobserved outcomes as well, respectively. Since generalized estimating equations (GEE) models, which provide valid inferences under MCAR mechanism, and likelihood-based models under MAR mechanism, are reasonably straightforward, many authors restrict modeling to 
these settings. However, when the missingness mechanism depends on the unobserved outcomes, these two procedures are likely to produce biased estimates of the measurement process parameters. To overcome this difficulty, many authors have proposed a model that incorporates both the information from the measurement process and the missing data process into a unified likelihood function (see, for example, Diggle and Kenward 1994; Molenberghs et al. 1997). This has provoked a large debate about the role of such models (see, for example, Scharfstein et al. 1999; Rotnitzky et al. 2001; Kenward et al. 2001 and Verbeke et al. 2001). The original enthusiasm was followed by skepticism about the strong and untestable assumptions on which this type of model rests. Models that incorporate the measurement and dropout processes are usually unidentifiable from observed data.

The goal of this paper is to use non-ignorable models to develop a procedure to assess the significance of local influence on model parameter estimates. To achieve this aim, we assume a class of joint likelihood models for the measurement and dropout processes and make use of this class to assess the impact of perturbations of the ignorable model in the direction of non-ignorable models. Specifically, we first use the concept of threshold crossing models to derive the joint distribution of bivariate ordinal outcomes, and then extend the model to allow for longitudinal data. Second, we formulate a model for the dropout process, where its dependence on the outcome process, potentially missing, is captured using the selection model of Diggle and Kenward (1994) on the latent scale. We show that this dropout selection model on the latent scale results in a shared random effects model between the measurement outcomes and the dropout process. This idea of using a shared latent variable model for analyzing longitudinal outcomes subject to informative dropouts is certainly not new (see, for example, Wu and Carroll 1988; Albert and Follmann 2000; Ten Have et al. 2002). Our extension of this technique is to allow an unspecified random effects distribution to add flexibility in the model. A well known alternative to this family of models is that of Scharfstein et al. (1999), which is based on the GEE principle. The GEE approach has several advantages over a fully parametric model. It is computationally tractable in applications where parametric approaches are computationally very demanding, if not impossible. Most importantly, likelihood-based models can be highly sensitive to any model misspecification that appears either in the measurement process or the dropout process. However, as reported by Little and Rubin (1999), GEE-based non-response models are confined to bias adjustment, whereas likelihood-based models have the potential to reduce both the bias and variance.

The proposed working model is described in Sect. 2; Sect. 3 discusses how graphical and analytical techniques can be used to assess a local sensitivity of the measurement parameter estimates under small perturbations of the ignorable model. Data from the Fluvoxamine study are used to illustrate the method in Sect. 4. Some remaining issues are discussed in Sect. 5.

\section{Description of the method}

\subsection{The measurement model}

It is conceptually convenient as noted by Ashford and Sowden (1970) to assume that the observed ordinal responses $Y=\left(Y_{1}, Y_{2}\right)^{\prime}$ are generated from an underlying 
unobservable latent variable $W=\left(W_{1}, W_{2}\right)^{\prime}$ with two sets of threshold values $a_{1}=\left(a_{11}, a_{12}, \ldots, a_{1, c_{1}-1}\right)^{\prime}$ and $a_{2}=\left(a_{21}, a_{22}, \ldots, a_{2, c_{2}-1}\right)^{\prime}$, where $c_{1}$ and $c_{2}$ represent the number of ordered levels for the first and the second marginal outcome, respectively. Specifically, the univariate responses (observed or missing) $Y_{1 \mathrm{kt}}$ and $Y_{2 k t}$ for unit $k=1, \ldots, K$, e.g., subject in the longitudinal setting and cluster in the repeated data setting, at time point $t=1, \ldots, n$, with $n$ being the number of planned time points, fall in category $i$ and $j$, respectively, if the first component $W_{1 k t}$ of the latent response exceeds $a_{1, i-1}$ but does not exceed $a_{1 i}$, and so for the second component $W_{2 k t}$ with respect to $a_{2, j-1}$ and $a_{2 j}$. The threshold values must be monotonically increasing to reflect the ordinal nature of the outcomes. For a binary outcome, only one threshold value representing the usual intercept is needed. By letting $a_{10}=a_{20}=-\infty$ and $a_{1 c_{1}}=a_{2 c_{2}}=\infty$, the threshold crossing model is formulated as follows,

$$
\left\{\begin{array}{l}
\left(Y_{1 k t}=i, Y_{2 k t}=j\right) \Leftrightarrow\left(a_{1, i-1} \leq W_{1 k t}<a_{1 i}, a_{2, j-1} \leq W_{2 k t}<a_{2 j}\right) \\
\forall i, j 1 \leq i \leq c_{1} \text { and } 1 \leq j \leq c_{2} .
\end{array}\right.
$$

Next, we consider a linear mixed effects regression model for the bivariate latent variable as follows,

$$
W_{\ell k t}=x_{\ell k t}^{\prime} \beta_{\ell}+z_{\ell k t}^{\prime} b_{\ell k}+\varepsilon_{\ell k t}, \quad \ell=1,2 .
$$

To complete this mixed model formulation, we assume that the random effects $b_{k}=\left(b_{1 k}^{\prime}, b_{2 k}^{\prime}\right)^{\prime}$ are generated from an unspecified distribution $\mathcal{F}$ and are independent of the residuals vector $\varepsilon_{k t}=\left(\varepsilon_{1 k t}, \varepsilon_{2 k t}\right)^{\prime}$. The column vectors $x_{1 k t}$ and $x_{2 k t}$, respectively of dimension $p_{1}$ and $p_{2}$, are the fixed effects design vectors with associated (column) vector of slopes $\beta_{1}$ and $\beta_{2}$. Similarly, $z_{1 k t}$ and $z_{2 k t}$ are the $r_{1} \times 1$ and $r_{2} \times 1$ design (column) vectors for the random effects associated with the column vectors of unknown random effects $b_{1 k}$ and $b_{2 k}$, respectively. The parameters $\beta_{1}$ and $\beta_{2}$ are common to all subjects, while $b_{1 k}$ and $b_{2 k}$ are subject-specific. For subject $k$, the vectors $x_{\ell k}=\left(x_{\ell k 1}, \ldots, x_{\ell k n}\right)^{\prime}$ and $z_{\ell k}=\left(z_{\ell k 1}, \ldots, z_{\ell k n}\right)^{\prime}, \ell=1,2$, often contain time-independent subject-specific covariates, but time-varying covariates are also allowed as indicated by Lesaffre et al. (2000) and Todem et al. 2002. To obtain a well-formulated model such as in Morell et al. (1997), we set the restriction that $\operatorname{rank}\left(x_{\ell k} \mid z_{\ell k}\right)=\operatorname{rank}\left(x_{\ell k}\right)$, for $\ell=1,2$, where $\left(x_{\ell k} \mid z_{\ell k}\right)$ represents the matrix obtained by combining the matrices $x_{\ell k}$ and $z_{\ell k}$.

For a bivariate mixed effects probit model, since individual variances of the error vector are not estimable from observed data (see, for example, Catalano and Ryan 1992), $\varepsilon_{k t}$ is distributed as a standardized bivariate normal distribution with mean 0 and correlation $\rho_{k t}$. The correlation coefficient $\rho_{k t}$ of the error terms $\varepsilon_{1 k t}$ and $\varepsilon_{2 k t}$ may be allowed to depend on covariates, with a design (column) vector $x_{\rho k t}$ and corresponding slope vector $\tau$. Specifically, this correlation coefficient can be modeled using the Fisher transformation,

$$
\log \left(\frac{1+\rho_{k t}}{1-\rho_{k t}}\right)=x_{\rho k t}^{\prime} \tau
$$

to ensure that $-1 \leq \rho_{k t} \leq 1$. 
Alternatively, if a bivariate logistic model is assumed, then the latent variable $W$ follows a bivariate Plackett (1965) distribution. Basically, the model uses the concept of global cross-ratios of Dale (1986) to represent the contemporaneous association and random effects terms for the longitudinal association (Ten Have and Morabia 1999).

\subsection{Dropout selection model}

Let denote by $R_{k t}=1$ if the response $Y_{k t}$ of subject $k$ at time point $t$ is observed and 0 if otherwise. If subject $k$ drops out at time $t_{k}$, therefore, the series of dropout indicator is given by $\mathcal{D}_{k, t_{k}}=\left\{R_{k 1}=\cdots=R_{k, t_{k}-1}=1, R_{k t}=0, R_{k, t_{k}+1}=\cdots=R_{k, n}=0\right\}$, otherwise if the subject completes the study, the dropout series is given by $\mathcal{D}_{k, n+1}=\left\{R_{k 1}=\cdots=R_{k, n}=1\right\}$. Since the dropout indicator $R$ is binary, we can assume the existence of an underlying continuous latent variable $W_{3}$ to represent this process. We can then formulate a model which allows the dropout latent outcome $W_{3}$ to depend on the latent variables $W_{1}$ and $W_{2}$ as follows,

$$
W_{3 k t}=x_{d k t}^{\prime} \beta_{d}+\delta_{p 1} W_{1 k, t-1}+\delta_{c 1} W_{1 k t}+\delta_{p 2} W_{2 k, t-1}+\delta_{c 2} W_{2 k t}+\varepsilon_{3 k t}
$$

where $x_{d k t}$ is the fixed effects design vector associated to the slope $\beta_{d}$ and $\varepsilon_{3 k t}$ is the measurement error. The parameters $\delta_{p 1}$ and $\delta_{p 2}$ are scalars associated with the previous latent variables $W_{1 k, t-1}$ and $W_{2 k, t-1}$, respectively, whereas $\delta_{c 1}$ and $\delta_{c 2}$ are associated with the current latent variables $W_{1 k t}$ and $W_{2 k t}$, respectively. In the dropout selection model of Molenberghs et al. (1997) and Ekholm et al. (2003), the latent variable $\left(W_{1}, W_{2}\right)$ is replaced with the observed outcome $\left(Y_{1}, Y_{2}\right)$. From the expression in (2) the dropout latent model in (3) can be rewritten as,

$$
W_{3 k t}=x_{3 k t}^{\prime} \beta_{3}+\lambda_{1}^{\prime} b_{1 k}+\lambda_{2}^{\prime} b_{2 k}+\varepsilon_{3 k t}
$$

where $x_{3 k t}$ is the fixed effects design vector associated to vector slope $\beta_{3}$ and the vectors $\lambda_{1}$ and $\lambda_{2}$ are associated with the vectors of unknown random effects $b_{1 k}$ and $b_{2 k}$, respectively. The variance of the error $\varepsilon_{3 k t}$ is not estimable from data at hand, and is therefore fixed to unity. Hence for a probit model, $\varepsilon_{3 k t}$ is assumed to be distributed as a standard normal with a cumulative distribution function $\Phi$. Let $T_{k}=1+\sum_{t=1}^{n} R_{k t}$ with $R_{k 1}=1$ be the dropout time for the $k$ th subject. The conditional instantaneous dropout probability at time $t$ is therefore modeled as,

$$
\operatorname{pr}\left(T_{k}=t \mid T_{k}>t-1, b_{k}\right)=\operatorname{pr}\left(W_{3 k t}<0 \mid b_{k}\right) .
$$

The graph in Fig. 1 depicts the relationship between the dropout and the measurement processes. The two processes are correlated since they share a common $b_{k}$. This formulation then induces informative dropout into the bivariate ordinal outcome $\left(Y_{1}, Y_{2}\right)$, whereby $T_{k}$ provides information about the $b_{k}$. Large positive values of $\lambda_{1}$ (or $\lambda_{2}$ ) correspond to the situation in which lower levels of the outcome $Y_{1}$ (or $Y_{2}$ ) drop out from the study prematurely.

\subsection{Model identifiability}

It is well known that non-ignorable models are typically not identifiable from observed data (Scharfstein et al. 1999). In particular for the non-ignorable model 


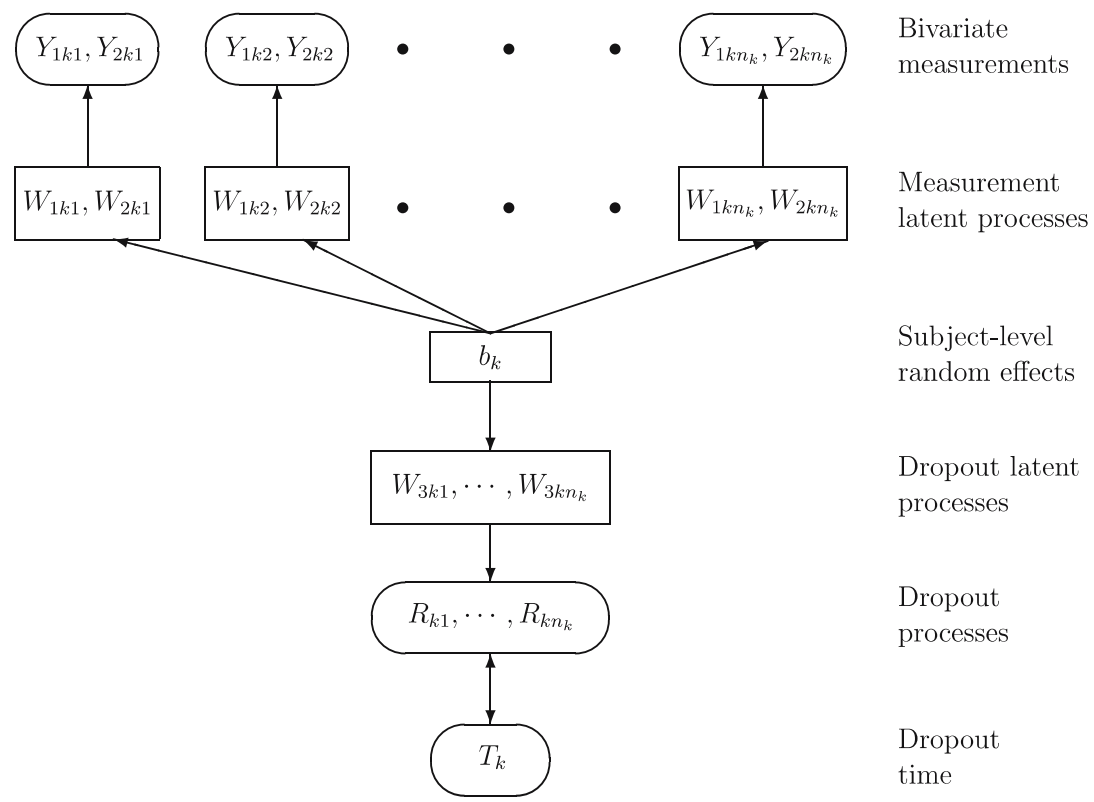

Fig. 1 Graph of the hierarchy of the measurement outcomes and latent processes with dropout component and random effects

described here, the random effects distribution, which serves as a mixing distribution, is not marginally estimable from observed data without additional assumptions. To see this, let $\mathcal{F}_{o}=\mathcal{L}(b \mid T>n)$ and $\mathcal{F}_{d}=\mathcal{L}(b \mid T \leq n)$ be the laws of the random effects $b$ for uncensored and censored subjects, respectively. Here, $\mathcal{L}$ stands for law and we have suppressed the subject index for simplicity. The marginal law of the random effects is given by

$$
\mathcal{F}=\left(1-p_{d}\right) \mathcal{F}_{o}+p_{d} \mathcal{F}_{d}
$$

where $p_{d}=P(T \leq n)$. If many independent copies of $I(T \leq n)$, where $I($.$) is the$ indicator function, are available, $p_{d}$ can be estimated very well. Although the random effects are not observed, the law $\mathcal{F}_{o}$ can also be well estimated via the law of the observed uncensored data. This is based on the fundamental assumption of conditional independence of responses for a given random effects level. A typical estimate of $\mathcal{F}_{o}$ is the non-parametric maximum likelihood estimate (MLE), which is a discrete distribution with support on at most $K-K_{d}$ points, where $K_{d}=\sum_{k} I\left(T_{k} \leq n\right)$ is the number of censored subjects (Lindsay 1983; Davidian and Giltinan 1998). The mystery is clearly $\mathcal{F}_{d}$, the law of $b$ for dropout subjects, which is not identifiable from observed data. The non-ignorable model as formulated above is therefore overspecified. Such over-specification can be managed in a more general way by considering a minimal set of parameters, conditional upon which the others are identifiable. It is important, however, to realize that the choice of the sensitivity parameter is non-unique and can be a difficult task (Kenward et al. 2001). There is a general consensus from the literature that, sensitivity parameters should be chosen from parameters that are not of primary interest. For our working selection model, a 
good choice would be characteristics that directly relate the measurement and the dropout processes. This translates into choosing $\mathcal{F}$ the law of the latent variable or $\lambda_{1}$ and $\lambda_{2}$ as our unidentifiable sensitivity terms. Choosing $\mathcal{F}$ as our sensitivity term is practically unfeasible, unless restricted to a very small class of distributions. We can avoid this problem by fixing both $\lambda_{1}$ and $\lambda_{2}$ and by estimating, possibly non-parametrically, the random effects distribution. An advantage of this approach is that it does not introduce possibly inappropriate and unverifiable assumptions about the distributions of random effects. We adopt and extend the predictive recursion (PR) for density estimation of the mixing distribution developed by Newton and Zhang (1999) in the univariate setup, to the multivariate context. This estimation method requires that the support of the mixing distribution be continuous and bounded.

\subsection{The PR estimation}

In this section, we estimate the random effects distribution using the PR estimation (Newton and Zhang 1999). For this, we assume that the measurement response and the dropout process are conditionally independent given random effects $b$. Let $f$ be the probability density function of the law $\mathcal{F}$ with respect to Lebesgue measure $\mathrm{d} b$ and let denote by $y_{k, 1:\left(t_{k}-1\right)}^{\text {obs }}$ the collection of all observed bivariate outcomes for subject $k$. The function $f($.$) is estimated by f^{K}($.$) , the final iteration step of the$ following recursive equation,

$$
f^{k}(b)=\left(1-\varpi_{k}\right) f^{k-1}(b)+\varpi_{k} \frac{f^{k-1}(b) \mathcal{L}\left(y_{k, 1:\left(t_{k}-1\right)}^{\text {obs }} \mid b\right) \mathcal{L}\left(t_{k} \mid b\right)}{\eta_{k}}, \quad k=1, \ldots, K,
$$

where $f^{0}(b)$ is the prior density, $\mathcal{L}\left(y_{k, 1:\left(t_{k}-1\right)}^{\text {obs }} \mid b_{k}\right)$ and $\mathcal{L}\left(t_{k} \mid b_{k}\right)$ are the conditional likelihood for the observed bivariate ordinal endpoints $y_{k, 1:\left(t_{k}-1\right)}^{\text {obs }}$ and the discrete dropout time $t_{k}$, respectively. The weight $\varpi_{k}$ is decreasing on the index range and $\eta_{k}=\int_{b} f^{k-1}(b) \mathcal{L}\left(y_{k, 1:\left(t_{k}-1\right)}^{\text {obs }} \mid b\right) \mathrm{d} b$ is a normalizing constant. The second term on the right of (6) is the Bayesian posterior density function of $b$ given observations from the $k$ th subject, with the prior density $f^{k-1}(b)$. Hence, the PR algorithm averages the density estimate from the previous step and the posterior density given the current observation. Although the final estimate $f^{K}($.$) depends on the order by which$ observations are processed, this dependence may be weak and often can be ignored (Tao et al. 1999). A typical choice for the weight is $\varpi_{k}=1 /(1+k)^{v}$, for which $v$ influences the amount of smoothing. Hence, a choice of $v$ close to 1 leads to a posterior distribution less influenced by individual observations and hence close to the prior. The prior density is typically chosen flat on a grid that should support most of the distribution. Tao et al. (1999) showed in a simulation study that estimation properties are insensitive to the choice of the prior. A key feature of (6) is that an approximation to a Dirichlet process posterior is produced in $O(K)$ steps (Newton et al. 1998). Newton and Zhang (1999) proved the consistency of the PR algorithm under certain regularity conditions for estimating a survival function with interval censoring.

To estimate this semiparametric model, we use a quasi-likelihood technique. Specifically, we first estimate the non-parametric component of the model, here the random effects distribution, and then supply its estimate into the likelihood for the parametric component to be estimated. Inverting the observed information matrix using this profile likelihood is known to be biased, however. Some adjustments then 
need to be made in order to reduce the bias to $O(1 / K)$ (McCullagh and Tibshirani 1990). We use the bootstrap method to estimate the standard errors of the model estimates (Efron and Tibshirani 1993). The method consists of randomly selecting $B$ samples of size $K$ with replacement from the original dataset. The sample standard deviations of the parameter estimates for the $B$ bootstrap samples estimate the true standard errors.

\subsection{Likelihood specification}

For a subject $k$, we denote by $y_{k, t_{k}: n}^{\text {mis }}$ the collection of all missing bivariate outcomes from the dropout time $t_{k}$ to the last pre-specified time point $n$, and by $Y_{k, t: t^{\prime}}$ the collection of all random vectors, from the $t$ th time point to the $t^{\prime}$ th time point, with $t \leq t^{\prime}$. Assuming that the measurement response and the dropout process are conditionally independent given random effects $b_{k}$, the contribution of a dropout subject $k$ to the likelihood function based on observed data $\left(y_{k, 1:\left(t_{k}-1\right)}^{\text {obs }}, t_{k}\right)$ is given by

$$
\begin{aligned}
\mathcal{L}\left(y_{k, 1:\left(t_{k}-1\right)}^{\mathrm{obs}}, t_{k}\right)= & \int_{b_{k}} \sum_{y_{k, t_{k}: n}^{\text {mis }}} \operatorname{pr}\left(Y_{k, 1:\left(t_{k}-1\right)}=y_{k, 1:\left(t_{k}-1\right)}^{\mathrm{obs}}, Y_{k, t_{k}: n}=y_{k, t_{k}: n}^{\text {mis }}, T_{k}=t_{k} \mid b_{k}\right) f\left(b_{k}\right) \mathrm{d} b_{k} \\
= & \int_{b_{k}} \sum_{y_{k, t_{k}: n}^{\text {mis }}} \operatorname{pr}\left(Y_{k, 1:\left(t_{k}-1\right)}=y_{k, 1:\left(t_{k}-1\right)}^{\mathrm{obs}}, Y_{k, t_{k}: n}=y_{k, t_{k}: n}^{\text {mis }} \mid b_{k}\right) \\
& \times \operatorname{pr}\left(T_{k}=t_{k} \mid b_{k}\right) f\left(b_{k}\right) \mathrm{d} b_{k} .
\end{aligned}
$$

The likelihood contribution for a censored subject then becomes,

$$
\mathcal{L}\left(y_{k, 1:\left(t_{k}-1\right)}^{\mathrm{obs}}, t_{k}\right)=\int_{b_{k}} \operatorname{pr}\left(Y_{k, 1:\left(t_{k}-1\right)}=y_{k, 1:\left(t_{k}-1\right)}^{\mathrm{obs}} \mid b_{k}\right) \operatorname{pr}\left(T_{k}=t_{k} \mid b_{k}\right) f\left(b_{k}\right) \mathrm{d} b_{k} .
$$

If a full-sequence data is observed, the contribution to the likelihood based on the data $\left(y_{k, 1: n}^{\text {obs }}, n+1\right)$ is given by

$$
\mathcal{L}\left(y_{k, 1: n}^{\mathrm{obs}}, n+1\right)=\int_{b_{k}} \operatorname{pr}\left(Y_{k, 1: n}=y_{k, 1: n}^{\mathrm{obs}} \mid b_{k}\right) \operatorname{pr}\left(T_{k}=n+1 \mid b_{k}\right) f\left(b_{k}\right) \mathrm{d} b_{k}
$$

The conditional dropout probability distribution is given by

$$
\operatorname{pr}\left(T_{k}=t_{k} \mid b_{k}\right)= \begin{cases}h_{k 2} & \text { if } t_{k}=2 \\ {\left[\prod_{t=2}^{t_{k}-1}\left(1-h_{k t}\right)\right] h_{k t}^{I\left(t_{k}<1+n\right)}} & \text { if } t_{k}=3, \ldots, n+1,\end{cases}
$$

where $h_{k t}=\operatorname{pr}\left(T_{k}=t \mid T_{k}-1, b_{k}\right)=1-\Phi\left(x_{3 k t}^{\prime} \beta_{3}+\lambda_{1}^{\prime} b_{1 k}+\lambda_{2}^{\prime} b_{2 k}\right)$ is the discrete hazard function. Given the random effects $b_{k}$, we assume that the bivariate ordinal outcome follows a multinomial distribution,

$$
\mathcal{L}\left(y_{k, 1: t_{k}}^{\mathrm{obs}} \mid b_{k}\right)=\prod_{t=1}^{t_{k}-1} \prod_{i=1}^{c_{1}} \prod_{j=1}^{c_{2}}\left[\operatorname{pr}\left(Y_{1 k t}=i, Y_{2 k t}=j \mid b_{k}\right)\right]^{I\left(y_{1 k t}^{\mathrm{obs}}=i\right) \times I\left(y_{2 k t}^{\mathrm{obs}}=j\right)} .
$$


Let $\alpha$ be the parameter vector of the measurement process including the smoothing parameter $v$ and let $\lambda=\left(\lambda_{1}^{\prime}, \lambda_{2}^{\prime}\right)^{\prime}$. The non-ignorable log-likelihood based on the observed ordinal outcomes $y_{k, 1:\left(t_{k}-1\right)}^{\text {obs }}, k=1, \ldots, K$, and the discrete dropout time $t_{k}=2, \ldots, n+1$, is given by

$$
L\left(\alpha, \beta_{3}, \lambda\right)=\sum_{k=1}^{K} \log \mathcal{L}\left(y_{k, 1:\left(t_{k}-1\right)}^{\mathrm{obs}}, t_{k}\right)=\sum_{k=1}^{K} \sum_{t=2}^{n+1} I\left(t_{k}=t\right) \log \mathcal{L}\left(y_{k, 1:(t-1)}^{\text {obs }}, t\right)
$$

As discussed in Appendix 1, the integration with respect to the random effects $b_{k}$ is done using either the Gauss-Legendre quadrature method or the Monte Carlo approach, depending on the dimension of the integration. We also discussed in Appendix 2 the optimization of the profile log-likelihood. Although the nonignorable log-likelihood is a continuous function of $\left(\alpha, \beta_{3}\right)$, for fixed $\lambda$, it involves recursive functions. Thus, maximizing the log-likelihood with respect to $\left(\alpha, \beta_{3}\right)$ using a Newton-Raphson approach with analytical derivatives is proved to be a difficult task. We used the Davidon-Fletcher-Powell (DFP) method, which only requires evaluations of the objective function to search for an optimum point (Brent 1972).

\section{Sensitivity analysis}

Estimating the non-ignorable model using the likelihood function specified in (7) is problematic. We then fix some model parameters and make use of the resulting nonignorable model to assess the sensitivity (locally) of the estimates around the ignorable model. Cook (1986) suggests that more confidence be put in a model that is relatively stable under small modifications. This can be achieved using both graphical and analytical techniques, which we review below.

\subsection{Graphical techniques for assessing sensitivity}

As our non-ignorable model is fundamentally non-identifiable, inference is possible only when unverifiable assumptions are made. We can set the vector $\left(\lambda_{1}, \lambda_{2}\right)$ to a known quantity in a compact set containing the point $\left(\lambda_{1}, \lambda_{2}\right)=(0,0)$ and estimate the remaining model parameters of the non-ignorable model. We denote by $\Theta$ the parameter vector $\left(\alpha^{\prime}, \beta_{3}^{\prime}\right)^{\prime}$ for a fixed $\lambda \neq 0$ and by $\widehat{\Theta}$ the corresponding MLE. Similarly, we denote by $\widehat{\Theta}_{0}$ the MLE of $\Theta_{0}$, for $\lambda=0$. Cook (1986) has proposed the so-called likelihood displacement defined by $-2\left\{L(\widehat{\Theta}, \lambda)-L\left(\widehat{\Theta}_{0}, 0\right)\right\}$ to measure the distance between MLE estimates $\widehat{\Theta}$ and $\widehat{\Theta}_{0}$. As reported by Verbeke and Molenberghs (2000), the graph of the likelihood displacement can then give an indication on the variability of $\widehat{\Theta}_{0}$. Hence if $\left.L\left(\Theta_{0}, 0\right)\right\}$ is strongly curved at $\widehat{\Theta}_{0}$ in which case $\Theta_{0}$ is estimated with high precision, then the likelihood displacement will be large.

To assess the influence on individual model parameter estimates, each component of the estimated difference $\widehat{\Theta}-\widehat{\Theta}_{0}$ is plotted against all fixed values of the vector $\left(\lambda_{1}, \lambda_{2}\right)$ in the compact set defined by $\left|\lambda_{\ell u}\right| \leq 1$, for $u=1, \ldots, r_{\ell}$, with $\ell=1,2$. The local approach then compares the MLE for fixed $\left(\lambda_{1}, \lambda_{2}\right)$ to that of the ignorable model in view of standard errors. As suggested by Verbeke et al. (2001), different 
estimates between these two schemes suggest that inferences are highly sensitive to small perturbations of the ignorable model in the direction of non-random models.

\subsection{Analytical techniques for assessing sensitivity}

The influence diagnosis can be expressed analytically and can be decomposed into interpretable components, which yields additional insights. The key idea is to develop a tool for assessing sensitivity of the model estimates without explicitly fitting the non-ignorable model. This can be done by expanding the non-ignorable likelihood function around the MLE of the ignorable model and making use of basic notions in matrix algebra. A second-order Taylor expansion of the non-ignorable log-likelihood function $L(\Theta, \lambda)$ around the point $\left(\widehat{\Theta}_{0}, 0\right)$ is given by

$$
\begin{aligned}
L(\Theta, \lambda)= & L\left(\widehat{\boldsymbol{\Theta}}_{0}, 0\right)+\left(\Theta-\widehat{\boldsymbol{\Theta}}_{0}\right)^{\prime}\left(\frac{\partial L(\Theta, \lambda)}{\partial \Theta}\right)_{\left(\widehat{\Theta}_{0}, 0\right)}+\lambda^{\prime}\left(\frac{\partial L(\Theta, \lambda)}{\partial \lambda}\right)_{\left(\widehat{\Theta}_{0}, 0\right)} \\
& +\frac{1}{2}\left(\left(\Theta-\widehat{\boldsymbol{\Theta}}_{0}\right)^{\prime}, \lambda^{\prime}\right) H\left(\left(\Theta-\widehat{\boldsymbol{\Theta}}_{0}\right)^{\prime}, \lambda^{\prime}\right)^{\prime}+o_{p}(1)
\end{aligned}
$$

where $H=\left(\begin{array}{ll}H_{11} & H_{12} \\ H_{12}^{\prime} & H_{22}\end{array}\right)$ with $H_{11}=\left(\begin{array}{ll}\frac{\partial^{2} L(\Theta, \lambda)}{\partial \alpha \partial \alpha^{\prime}} & \frac{\partial^{2} L(\Theta, \lambda)}{\partial \alpha \partial \beta_{3}^{\prime}} \\ \frac{\partial^{2} L(\Theta, \lambda)}{\partial \beta_{3} \partial \alpha^{\prime}} & \frac{\partial^{2} L(\Theta, \lambda)}{\partial \beta_{3} \partial \beta_{3}^{\prime}}\end{array}\right)_{\left(\widehat{\Theta}_{0}, 0\right)}, H_{12}=\left(\frac{\partial^{2} L(\Theta, \lambda)}{\partial\left(\alpha, \beta_{3}\right) \partial \lambda^{\prime}}\right)_{\left(\widehat{\Theta}_{0}, 0\right)}$ and $H_{22}=\left(\frac{\partial^{2} L(\Theta, \lambda)}{\partial \lambda \partial \lambda^{\prime}}\right)_{\left(\widehat{\Theta}_{0}, 0\right)}$. Taking derivatives with respect to $\Theta$ on both sides of (8) gives,

$$
\frac{\partial L(\Theta, \lambda)}{\partial \Theta} \approx H_{11}\left(\Theta-\widehat{\Theta}_{0}\right)+H_{12} \lambda
$$

At the MLE $\widehat{\Theta}$ and for a fixed $\lambda$, we get, $\left(\frac{\partial L(\Theta, \lambda)}{\partial \Theta}\right)_{(\widehat{\Theta}, \lambda)}=0$ and therefore $H_{11}\left(\widehat{\Theta}-\widehat{\Theta}_{0}\right)+H_{12} \lambda=0$ which gives,

$$
\widehat{\Theta} \approx \widehat{\Theta}_{0}-H_{11}^{-1} H_{12} \lambda .
$$

Under regularity conditions, we have that $\left(\frac{\partial^{2} L(\Theta, \lambda)}{\partial \alpha \partial \beta_{3}^{\prime}}\right)_{\left(\widehat{\Theta}_{0}, 0\right)}=\left(\frac{\partial^{2} L(\Theta, 0)}{\partial \alpha \partial \beta_{3}^{\prime}}\right)_{\widehat{\Theta}_{0}}=0$ and similarly that $\left(\frac{\partial^{2} L(\Theta, 0)}{\partial \beta_{3} \partial \alpha^{\prime}}\right)_{\widehat{\Theta}_{0}}=0$. We finally get for the measurement model parameters,

$$
\widehat{\alpha} \approx \widehat{\alpha}_{0}-\left(\frac{\partial^{2} L(\Theta, 0)}{\partial \alpha \partial \alpha^{\prime}}\right)_{\widehat{\Theta}_{0}}^{-1}\left(\sum_{\ell=1,2}\left(\frac{\partial^{2} L(\Theta, \lambda)}{\partial \alpha \partial \lambda_{\ell}^{\prime}}\right)_{\left(\widehat{\Theta}_{0}, 0\right)} \times \lambda_{\ell}\right) .
$$

For any unit vector $\lambda$, we define a sensitivity index, $S_{\lambda}(\widehat{\alpha})$, of $\alpha$ with respect to $\lambda$ as follows,

$$
S_{\lambda}(\widehat{\alpha})=\left(\frac{\partial^{2} L(\Theta, 0)}{\partial \alpha \partial \alpha^{\prime}}\right)_{\widehat{\Theta}_{0}}^{-1} \sum_{\ell=1,2}\left(\frac{\partial^{2} L(\Theta, \lambda)}{\partial \alpha \partial \lambda_{\ell}^{\prime}}\right)_{\left(\widehat{\Theta}_{0}, 0\right)} \times \lambda_{\ell}, \quad \ell=1,2 .
$$

This sensitivity index can be computed for any direction $\lambda$. One evident choice is to consider the vector $\lambda$ for which the entries are all zero except for the entry $\lambda_{\ell u_{0}}$ fixed at unity for some $\ell$. In other words, we have $\lambda_{\ell u_{0}}=1$ and $\lambda_{\ell u}=0$ for some $\ell$, and all $u \neq u_{0}$. The corresponding sensitivity index with respect to $\lambda_{\ell u_{0}}$ is then given by 


$$
S_{\lambda_{\ell u_{0}}}(\widehat{\alpha})=\left(\frac{\partial^{2} L(\Theta, 0)}{\partial \alpha \partial \alpha^{\prime}}\right)_{\widehat{\Theta}_{0}}^{-1}\left(\frac{\partial^{2} L(\Theta, \lambda)}{\partial \alpha \partial \lambda_{\ell u_{0}}^{\prime}}\right)_{\left.\widehat{\Theta}_{0}, 0\right)} .
$$

Since $\widehat{\alpha}$, the estimate of the measurement parameter, is a function of $\lambda$, we write $\widehat{\alpha}(\lambda)$ to highlight this dependence. In addition, the estimate $\widehat{\alpha}(\lambda)$ is approximately linear in $\lambda$ from Eq. (10) and by using a first-order Taylor expansion of $\widehat{\alpha}(\lambda)$ around $\lambda=0$, we also have the following approximation,

$$
\widehat{\alpha}(\lambda) \approx \widehat{\alpha}(0)+\sum_{\ell=1,2} \lambda_{\ell}\left(\frac{\partial \widehat{\alpha}(\lambda)}{\partial \lambda_{\ell}}\right)_{\lambda=0} .
$$

Hence, an approximation to the sensitivity index with respect to $\lambda_{\ell u_{0}}$ is given by

$$
S_{\lambda_{\ell u_{0}}}(\widehat{\alpha}) \approx\left(\frac{\partial \widehat{\alpha}(\lambda)}{\partial \lambda_{\ell u_{0}}}\right)_{\lambda=0} \approx \widehat{\alpha}\left(\lambda_{\ell u_{0}}^{*}\right)-\widehat{\alpha}(0), \quad \ell=1,2,
$$

where $\lambda_{\ell u_{0}}^{*}=\left(0, \ldots, 0=\lambda_{\ell, u_{0}-1}, 1=\lambda_{\ell, u_{0}}, 0 \ldots, 0\right)^{\prime}$.

If the absolute values of elements of $S_{\lambda_{\ell u_{0}}}(\widehat{\alpha})$ are large, small changes in the direction of the parameter vector $\lambda_{\ell u_{0}}, \ell=1,2$, would lead to relatively large differences in model estimates (Ma, G. and Heitjan, D., unpublished manuscript). A question that quickly emerges is how large should $S_{\lambda_{\ell u_{0}}}(\widehat{\alpha})$ be for the conclusion to be drawn? This is a recurrent difficulty in establishing a threshold value above which a component of $\lambda$ is influential. One could classify a component $\lambda_{\ell u_{0}}$ as influential if the magnitude of a standardized version of $S_{\lambda_{\ell u_{0}}}(\widehat{\alpha})$ is greater than 2 . This implicitly performs the tests of difference between estimates when the ignorable model is perturbed in the direction $\lambda_{\ell u_{0}}$, using the large sample theory. An estimate of the standard error of components of $S_{\lambda_{\ell u_{0}}}(\widehat{\alpha})$ then needs to be computed. We note, however, that an explicit expression for the standard error of these sensitivity indexes is hard, if not impossible, to derive analytically. We therefore rely on the bootstrap technique for their approximation. The sample standard deviation of the estimates of the sensitivity parameter for $B$, say 1000 bootstrap samples, estimates the true standard error.

\section{Application to the Fluvoxamine study}

In Fig. 2, the evolution of side effects and the therapeutic response is depicted for all complete (full-sequence) and incomplete (dropout) cases. Compared to the start of the study, a substantial improvement of side effects and a tendency for the therapeutic effect to wear off are depicted over time, both among completers and noncompleters. Most importantly, the dropout mechanism appears to be linked, mostly, to the severity of side effects. Specifically, study non-completers are likely to be doing poorly with respect to observed side effects responses. This automatically rules out a MCAR mechanism. Several questions then emerge. How would inferences about the time evolution of side effects and the therapeutic response be altered by a potentially non-random dropout mechanism? In particular, how sensitive are the 


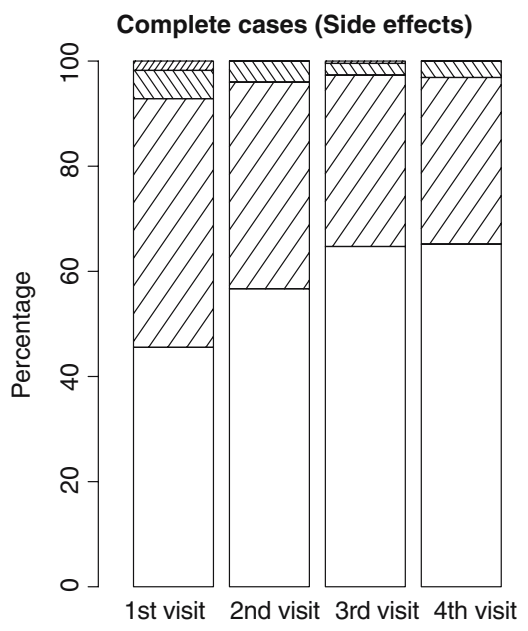

Dropout cases (Side effects)

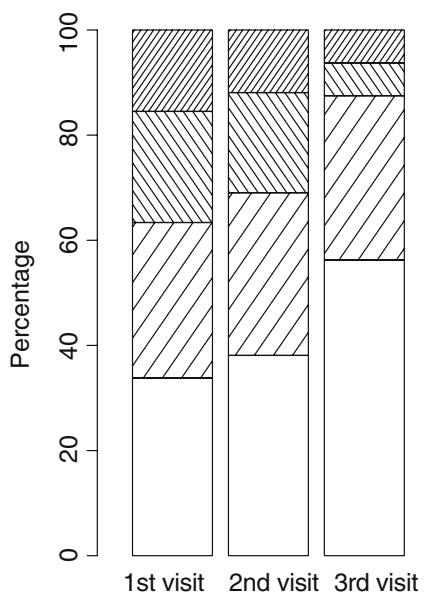

$$
\begin{aligned}
& \square(1) \text { no side effects } \\
& \square(2) \text { no significant side effects } \\
& \nabla(3) \text { significant side effects } \\
& \square(4) \text { side > therapeutic effects }
\end{aligned}
$$

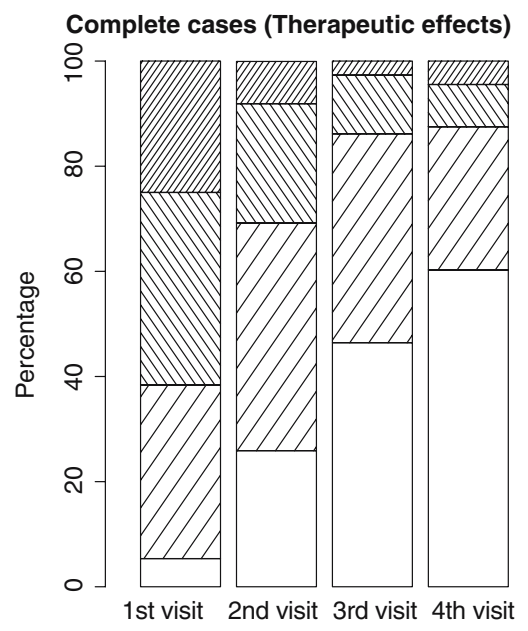

\section{Dropout cases (Therapeutic effects)}

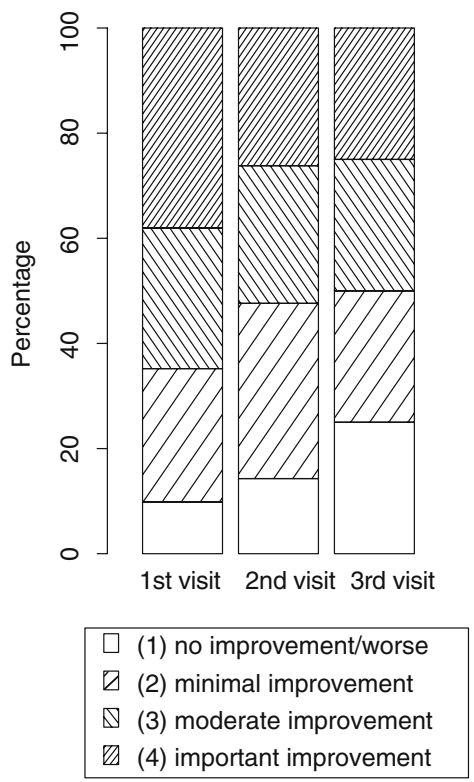

Fig. 2 Evolution of the side effects and the therapeutic response for all available and complete cases over the 4 follow-up visits. Available cases at visits 1, 2, 3 and 4 are 299, 268, 242 and 224, respectively and complete cases are 224 throughout the study

time effects estimates when the ignorable model is locally perturbed in the direction of non-random models?

As a preliminary analysis, a random intercept measurement model with an unstructured fixed effect of time and a probit link is fitted to the complete and incomplete dataset. As expected only two contrasts of time effects are estimated for censored subjects. The results of the models estimates are shown in Table 1 and are consistent with those of the bar charts presented in Fig. 2. In particular, side effects 
Table 1 Parameter estimates, (standard errors) and ( $P$-values $)^{*}$ of the mixed effects model fitted to the complete and incomplete cases of the Fluvoxamine dataset

\begin{tabular}{llcc}
\hline Component & Parameter & Complete cases & Incomplete cases \\
\hline Side effects & $a_{11}$ & $-1.19(0.24)$ & $-1.74(0.15)$ \\
& $a_{12}-a_{11}$ & $2.91(0.17)$ & $2.00(0.36)$ \\
& $a_{13}-a_{11}$ & $4.02(0.24)$ & $3.88(0.50)$ \\
& visit $2-$ visit 1 & $-0.56(0.16)$ & $0.17(0.28)(0.5390)$ \\
& visit $3-$ visit 1 & $-0.98(0.16)$ & $0.39(0.41)(0.3483)$ \\
Therapeutic effects & visit $4-$ visit 1 & $-0.97(0.16)$ & - \\
& $a_{21}$ & $-2.38(0.22)$ & $-1.35(0.52)(0.0090)$ \\
& $a_{22}-a_{21}$ & $1.74(0.10)$ & $2.17(0.41)$ \\
& $a_{23}-a_{21}$ & $3.06(0.13)$ & $3.46(0.46)$ \\
& visit $2-$ visit 1 & $-1.19(0.12)$ & $-0.50(0.27)(0.0575)$ \\
Cross-association & visit $3-$ visit 1 & $-2.06(0.14)$ & $-0.76(0.40)(0.0555)$ \\
PR smoother & $\operatorname{visit~} 4-$ visit 1 & $-2.39(0.15)$ & - \\
Summary measure & $\log \{(1+\rho) /(1-\rho)\}$ & $0.19(0.16)(0.2421)$ & $0.29(0.12)(0.0130)$ \\
& $\log \{v /(1-v)\}$ & $1.75(0.46)$ & $1.59(0.50)(0.0016)$ \\
\hline
\end{tabular}

*Non-reported $P$-values are less than 0.0001

decrease over time among completers, but tend to increase for non-completers although the effect is found to be nonsignificant. For the therapeutic response however, the time effects decline over time both for completers and non-completers, but the magnitude of the decline is higher among completers. Although subjects who dropped out prematurely from the study are likely to have a lower decline in therapeutic effects, this relative advantage is outweighed by more severe side-effects.

We further perform a sensitivity analysis based on the random subject effect on both measurement outcomes and discrete dropout model $h_{k t}=1-\Phi\left(\delta_{0}+\right.$ $\left.\lambda_{1} b_{1 k}+\lambda_{2} b_{2 k}\right)$. To assess local influence using a global approach, we plot the likelihood displacement $-2\left\{L(\widehat{\Theta}, \lambda)-L\left(\widehat{\Theta}_{0}, 0\right)\right\}$ by allowing the vector $\lambda$ to vary in the compact square defined by $\left|\lambda_{\ell}\right| \leq 1$ for $\ell=1,2$. The plot is shown in Fig. 3 . The graph appears to be strongly curved at $\lambda_{1}=\lambda_{2}=0$, which indicates that the nonignorable model is estimated with high precision. To assess the influence on individual model parameter estimates, we graphically represent elements of the difference, $\widehat{\alpha}(\lambda)-\widehat{\alpha}_{0}$, between estimate of the non-ignorable model for fixed values of $\lambda_{1}$ and $\lambda_{2}$ in the square $[-1,1] \times[-1,1]$ and that of the ignorable model. The plots of the contrast visit 2 - visit 1 are shown in Fig. 4 for the side effects and the therapeutic response. Plots of other effects indicated the same results and thus are not shown. As expected, time effects on the side effects appear to be more sensitive to $\lambda_{1}$, whereas its effects on the therapeutic effect appear to be more sensitive to $\lambda_{2}$. Cautions should, however, be taken in interpreting these plots as the standard errors are not accounted for. In addition, adding a $95 \%$ confidence region on the same plot may not be practical for visualization. Instead, we summarize the plot on two-dimensional spaces by selecting few points $\left(\lambda_{1}, \lambda_{2}\right)$ on the square and constructing confidence intervals around estimates of the corresponding time effects contrasts. The plots are shown in Fig. 5 for side effects and the therapeutic outcome. All nonignorable estimates lie within a $95 \%$ confidence interval of the ignorable parameter estimate. Therefore, perturbations of at most one unit of the ignorable model in the direction of the non-ignorable model have relatively little effects on the estimates. 


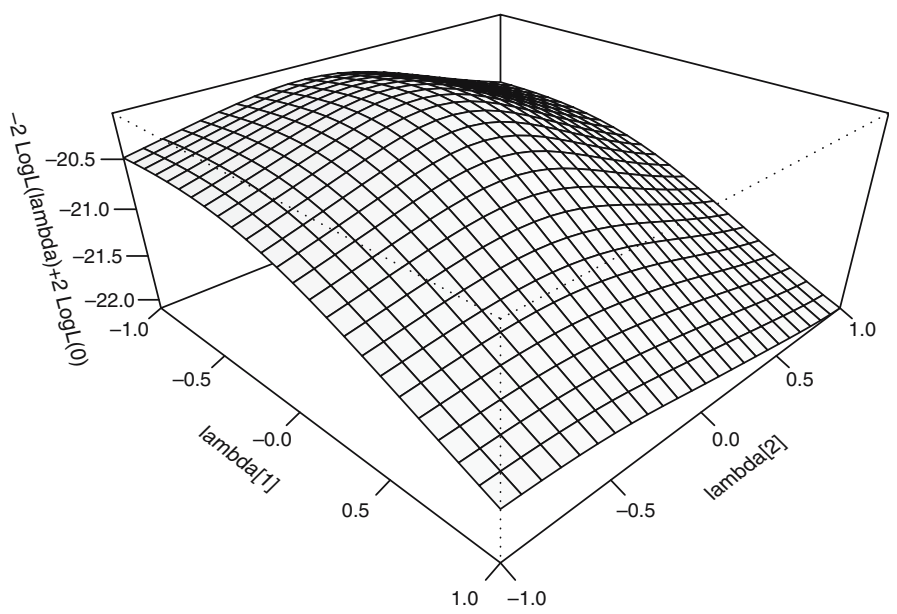

Fig. 3 Plot of $-2\left\{L(\widehat{\Theta}, \lambda)-L\left(\widehat{\Theta}_{0}, 0\right)\right\} / 100$ versus $\lambda_{1}$ and $\lambda_{2}$

Furthermore, to assess the sensitivity of the time effects on the side effects outcome with respect to $\lambda_{1}$, we set $\lambda_{2}=0$ and construct confidence intervals around the corresponding estimates of the model for values of $\lambda_{1}$ in the range [ $\left.-1,1\right]$. A similar plot is constructed for the therapeutic response and all plots are shown in Fig. 6. Estimates appear to be relatively stable under a perturbation of at most one unit of the ignorable model in the direction of non-ignorable model. All time effects estimates from the non-ignorable models fall within a $95 \%$ confidence interval of the corresponding estimates from the ignorable model. Therefore, despite the fact that the time effects on the therapeutic response appear to be more sensitive to $\lambda_{2}$, this influence is minor in the light of standard errors.

Applied to the Fluvoxamine data set, we computed the estimates of the sensitivity index for the time effects, parameters of primary interest. The results are shown in Table 2 and are consistent with those of the graphical technique discussed above. In particular, all standardized coefficients are lower than 2, suggesting that time effects are insensitive to small changes of the vector $\lambda$ overall. Despite this overall trend, the time effects on side effects (the therapeutic response) are more sensitive to $\lambda_{1}$ than $\lambda_{2}\left(\lambda_{2}\right.$ than $\left.\lambda_{1}\right)$. This should be expected from the model formation.

The evidence from our investigation speaks against dropout being at random in the Fluvoxamine data, but the sensitivity analysis results suggest that the model parameters are not much locally influenced by dropouts. The ignorable model is therefore preferable for its relative simplicity. As a final analysis, we then fit the ignorable model using all available data. We also include baseline information such as age, severity and duration of disease found to be predictive of side effects in previous studies (see, for, example, Molenberghs et al. 1997). The effects of baseline characteristics on the therapeutic outcome were also tested, but turned out to be non-significant. The result of the final model fit is given in Table 3 .

Hence, from a clinical and epidemiological standpoints, our analysis suggests that the intensity of side effects declines with time. Despite this overall performance of the drug from a safety standpoint, older patients and patients who had a long history of the disease at baseline, tend to have the worst severe side effects. There was also a tendency of the therapeutic effects to wear off over time. Finally, the estimate of the 


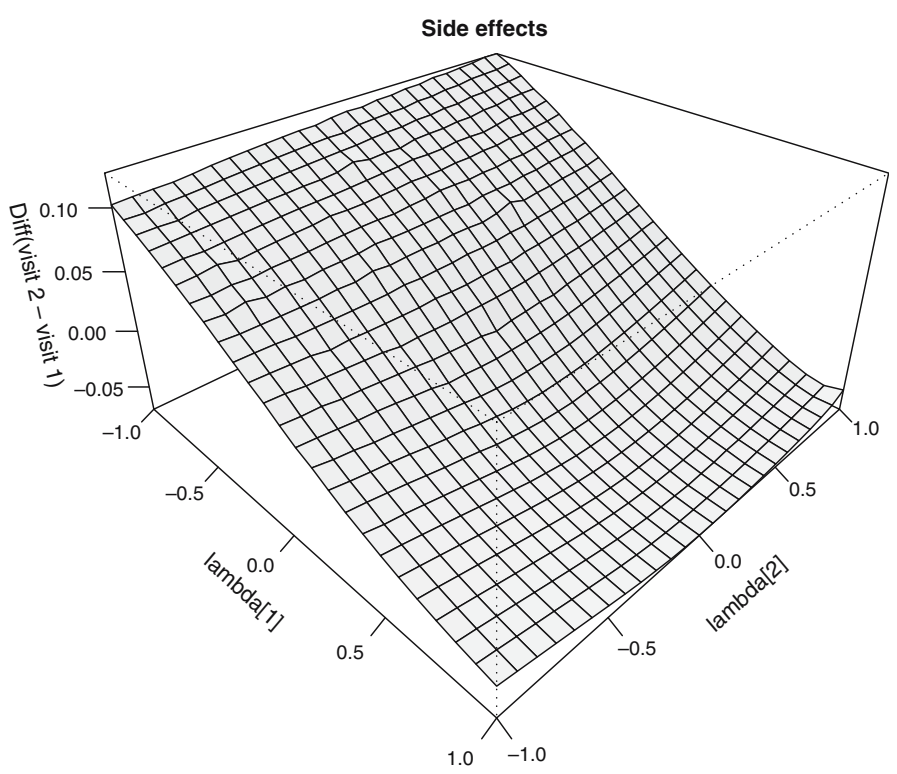

Therapeutic effects

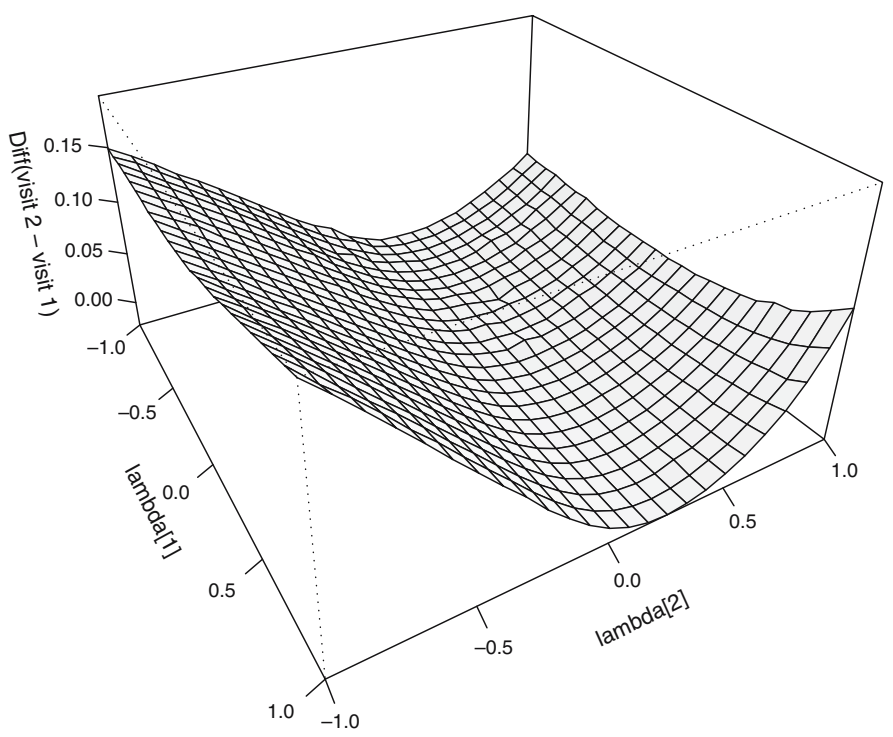

Fig. 4 Plot of the difference between (visit 2 - visit 1$)$ contrast for fixed $\left(\lambda_{1}, \lambda_{2}\right)$ and (visit 2 - visit 1 ) contrast for $\lambda=0$, both for the side effects and the therapeutic response

polychoric coefficient $\rho$ is positive suggesting that patients who have the most therapeutic benefits also have the worst side effects. Regarding the dropout profile, subjects who drop out are likely to have severe side effects although they also benefited most from the therapeutic effects. 


\section{Side effects}
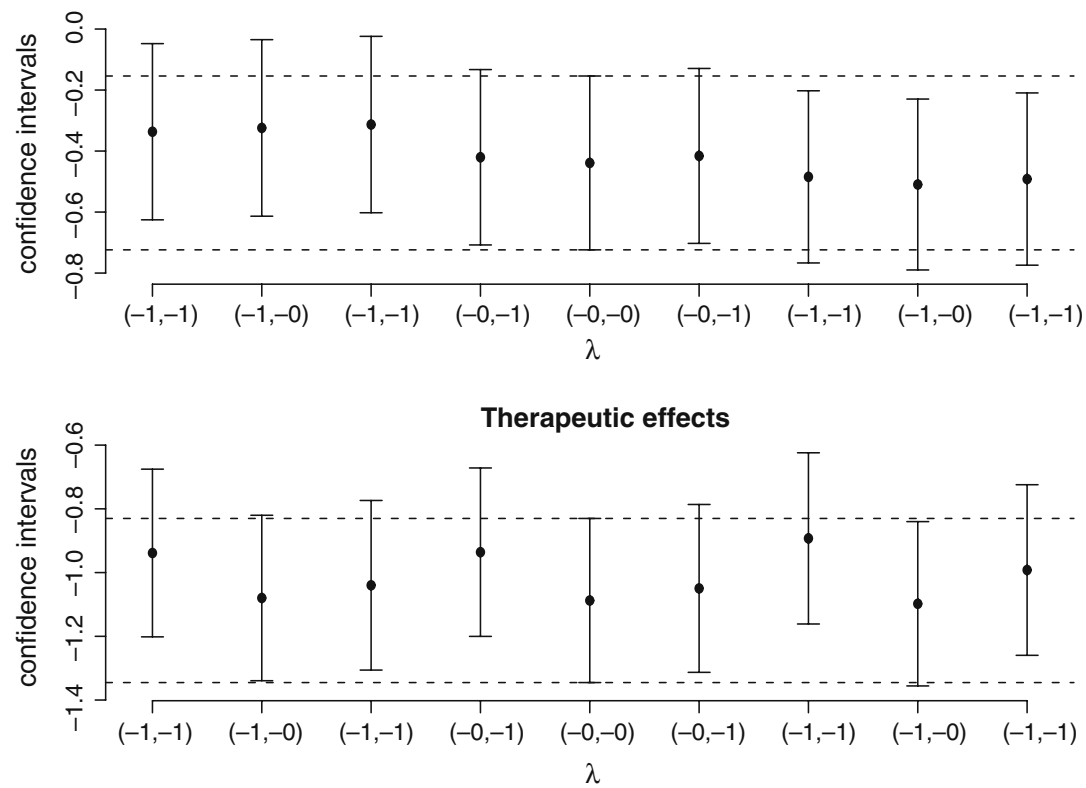

Fig. $595 \%$ confidence intervals of (visit 2 - visit 1 ) contrasts for selected points $\lambda=\left(\lambda_{1}, \lambda_{2}\right)$; and $95 \%$ lower and upper MAR confidence bounds for the side effects and therapeutic effects, respectively

The PR density is also estimated at the final iteration using the expression,

$$
f^{k}(b)=\left(1-\varpi_{k}\right) f^{k-1}(b)+\varpi_{k} \frac{f^{k-1}(b) \mathcal{L}\left(y_{k, 1:\left(t_{k}-1\right)}^{\mathrm{obs}} \mid b\right)}{\int_{b} f^{k-1}(b) \mathcal{L}\left(y_{k, 1:\left(t_{k}-1\right)}^{\mathrm{obs}} \mid b\right) \mathrm{d} b}, \quad k=1, \ldots, K,
$$

which is derived from (6) as the dropout likelihood $\mathcal{L}\left(t_{k} \mid b_{k}\right)$ does not depend on the random effect $b$. The density estimate is plotted in Fig. 7. This plot displays two peaks. To see if this bimodality is the result of chance, we selected bootstrap samples from the original and estimated the mixing distribution. Most of the corresponding PR density estimates also displayed the same peaks as the original sample, suggesting that the bimodality is not the result of chance. Further examination indicated that subjects who benefited most from the therapeutic effects and have more severe side effects contributed heavily to the higher mode of the mixing distribution. The lower mode is mostly made of subjects who did have mild or no severe side effects with little therapeutic benefits.

\section{Discussion}

The Fluvoxamine data set has been previously analyzed in other methodological work (see, for example, Lesaffre et al. 1996; Molenberghs et al. 1997; Ekholm et al. 2003). The important difference between our analysis and these previous work is that we jointly study the side effects and the therapeutic response together with the dropout mechanism. Previous work have concentrated only on the univariate aspect 


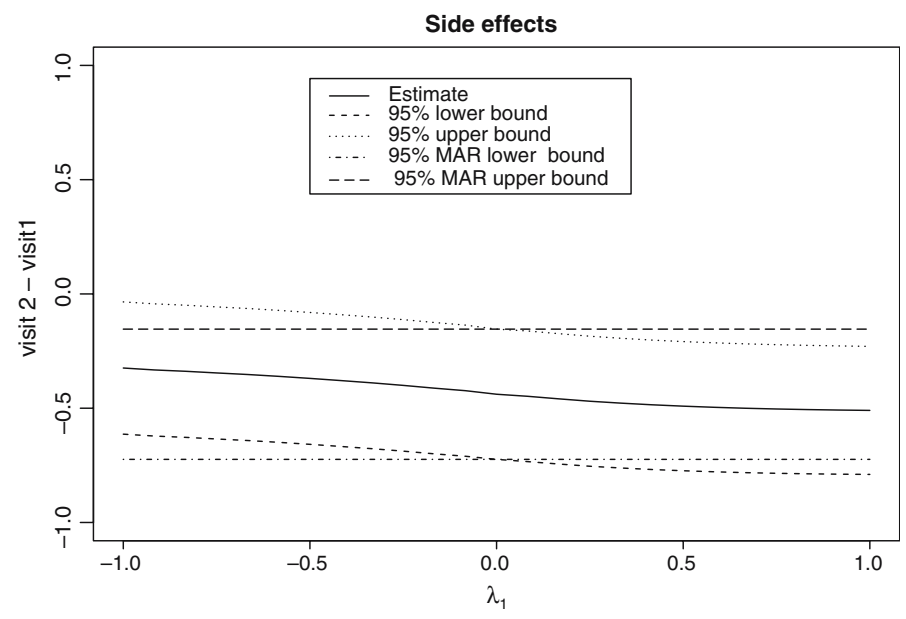

Therapeutic effects

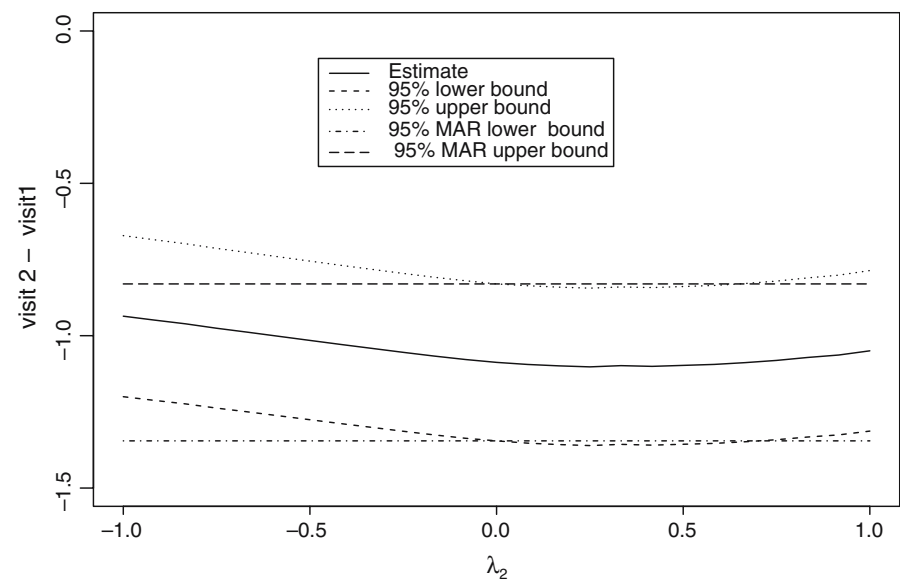

Fig. $695 \%$ confidence bounds of (visit 2 - visit 1 ) contrasts over the range of $\lambda_{2}$ (with $\lambda_{1}=0$ ) and $\lambda_{1}$ (with $\left.\lambda_{2}=0\right)$ for the side effects and therapeutic effects, respectively

Table 2 Standardized sensitivity index estimates for the random intercept mixed effects model fitted to the Fluvoxamine dataset

\begin{tabular}{llll}
\hline Component & Parameter & $S_{\lambda_{1}}(\widehat{\alpha})$ & $S_{\lambda_{2}}(\widehat{\alpha})$ \\
\hline Side effects & visit 2 - visit 1 & 0.54 & 0.08 \\
& visit 3 - visit 1 & 0.92 & 0.13 \\
Therapeutic effects & visit 4 - visit 1 & 0.99 & 0.01 \\
& visit 2 - visit 1 & 0.05 & 0.81 \\
& visit 3 - visit 1 & 0.07 & 1.43 \\
& visit 4 - visit 1 & 0.10 & 1.97 \\
\hline
\end{tabular}

of the problem and therefore give a partial view of the underlying true mechanism. Our approach attempts to comprehend the complexity of the underlying process that generates the two outcomes and dropouts. This is done by incorporating both the information from the measurement process and the dropout process into a unified likelihood model. We specifically assume a three-dimensional latent variable for 
Table 3 Parameter estimates, (standard errors) and $(P$ values) of the mixed effects model fitted to all available data ${ }^{*}$ Non-reported $P$-values are less than 0.0001

\begin{tabular}{lll}
\hline Component & Parameter & $\begin{array}{l}\text { Estimate } \\
(\text { s.e })(P \text {-value })^{*}\end{array}$ \\
\hline Side effects & $a_{11}$ & $-0.10(0.38)(0.7756)$ \\
& $a_{12}-a_{11}$ & $2.59(0.15)$ \\
& $a_{13}-a_{11}$ & $3.70(0.22)$ \\
& Age & $0.35(0.14)$ \\
& Duration & $0.28(0.08)$ \\
& Severity & $-0.26(0.10)$ \\
& visit $2-$ visit 1 & $-0.48(0.13)(0.0002)$ \\
Therapeutic & visit $3-$ visit 1 & $-0.90(0.14)$ \\
effects & visit $4-$ visit 1 & $-0.95(0.15)$ \\
& $a_{21}$ & $-2.21(0.23)$ \\
& $a_{22}-a_{21}$ & $1.71(0.10)$ \\
& $a_{23}-a_{21}$ & $2.98(0.14)$ \\
& visit $2-$ visit 1 & $-1.08(0.14)$ \\
Cross-association & $\operatorname{visit} 3-$ visit 1 & $-1.93(0.16)$ \\
PR smoother & $\log \{(1+\rho) /(1-\rho)\}$ & $0.29(0.18)(0.1078)$ \\
Summary measure & $\log \{v /(1-v)\}$ & $1.87(0.50)(0.0002)$ \\
& $-2 \log L$ & 3507.95 \\
& &
\end{tabular}

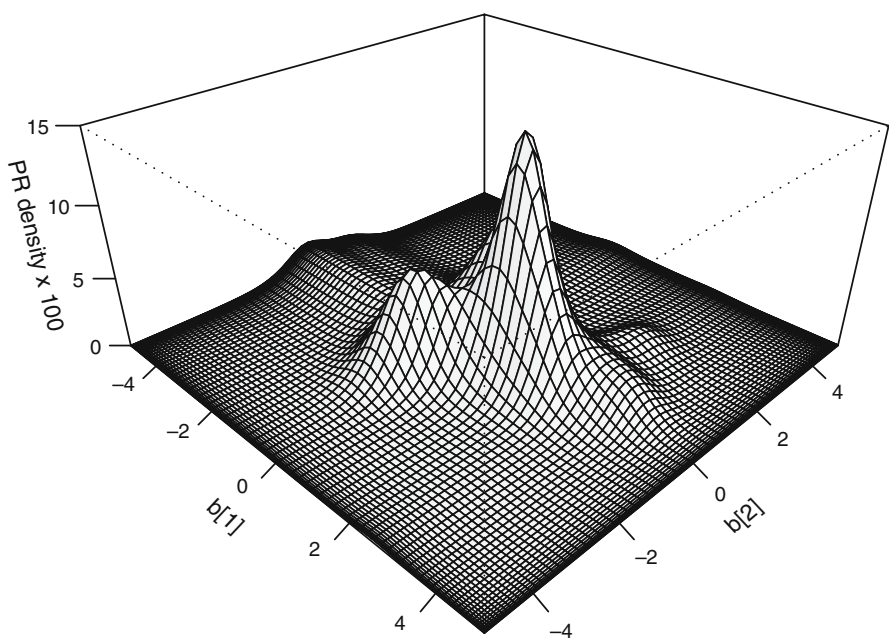

Fig. 7 PR density estimation of the mixing distribution using all available data

which the first two components are used to model the measurement process and the last component accounts for the dropout process. The dropout latent variable was constrained to depend on the measurement latent variables, which resulted in a shared non-ignorable random effects model. Estimating this non-ignorable model is problematic, however, because the numerical optimization can be difficult due to a flat likelihood and estimates can be sensitive to untestable assumptions. Such overspecification was managed in a more general way by considering a minimal set of parameters, conditional upon which the others are identifiable. The proposed class of models was then used to assess sensitivity (locally) of the estimates around the ignorable models using graphical and analytical techniques. For analytical techniques, a sensitivity index is constructed to assess effects of small perturbations of 
the ignorable model in the direction of non-ignorable models. We found particularly easy the calculation of such an index which uses only quantities derived from the ignorable model. This approach therefore helps avoid the need to fit non-ignorable models in assessing sensitivity as typically done in graphical techniques.

The results of this investigation can serve as a prototype for analyzing data from clinical trials on antidepressant drugs, which are typically known to generate many dropouts due to severe adverse outcomes. Our method is in general suitable for analyzing longitudinal studies where the primary endpoints are bivariate ordinal and dropout is non-ignorable or informative. An important strength of the method is that we estimate non-parametrically the random effects distribution using the PR method by Newton and Zhang (1999). This approach can help visualize the distribution of the data. For example, a distribution with multiple modes might indicate the presence of unknown risk groups unexplained by covariates under investigation. In this respect, this distribution estimate can also be used as a check of model fit (see, for, example, Tao et al. 1999). Moreover, unexpected risk scores on the random effects scale may point to erroneous functional relationships or differential measurement errors in the responses.

Several extensions of the proposed methodology are possible. First, the measurement model may be extended to accommodate more than two responses and the dropout process may also be extended to account for competing causes of censoring or dropout. Second, the method can be adapted to pattern mixture models and classical selection models not formulated as a shared random effects model. Finally, a sensitivity analysis can be made more globally by assuming that the non-identifiable parameter lies in the whole parameter space, not necessary the neighborhood of the MAR model parameters. This last issue is the focus of current work.

Acknowledgments The authors wish to thank Solvay Pharma B.V. for permission to use the data from the Fluvoxamine study.

\section{Appendix 1}

Numerical integration of the profile log-likelihood

In consideration of a bounded continuous mixing distribution, the Gauss-Legendre quadrature is chosen to approximate the integration. Reasonable lower and upper bounds of the integration can be obtained from the estimates of the standard deviation of a fully parametric normal model. Specifically, for each dimension of the random effects, the range of integration is chosen to be $\left(-5 S D_{\ell u}, 5 S D_{\ell u}\right)$, where $S D_{\ell u}$ is the estimated standard deviation of the $u$ th normal random effects of the $\ell$ th outcome. The corresponding product space approximately supports all probability mass of the mixing distribution. In confining the support of the random effects to a finite space, the starting density of the mixing distribution is chosen to be uniform given by

$$
f^{0}(b)=\prod_{\ell=1,2} \prod_{u=1}^{r_{\ell}} \frac{1}{10 S D_{\ell u}}, \quad \forall b,
$$

where $r_{\ell}$, for $\ell=1,2$ is dimension of the random effects design vector $z_{\ell}$. When the random effects dimension $r_{1}+r_{2}$ is large, say greater than 4 , a Monte Carlo simulation 
is used to perform the integration. It is widely known that for higher dimensional integrals, the number of support points in a quadrature approach increases exponentially compared to that of a Monte Carlo approach, which increases only linearly.

\section{Appendix 2}

Optimization of the profile log-likelihood for fixed $\lambda$

The marginal non-ignorable log-likelihood for a sample of $K$ independent subjects is given by

$$
L\left(\alpha, \beta_{3}, \lambda\right)=\sum_{k=1}^{K} \log \mathcal{L}\left(y_{k, 1:\left(t_{k}-1\right)}^{\text {obs }}, t_{k}\right) .
$$

Although this non-ignorable log-likelihood is a continuous function of $\left(\alpha, \beta_{3}\right)$ for fixed $\lambda$, it involves recursive functions. Specifically, the smoothing parameter $v$ of the mixing distribution is imbedded in recursive functions, which do not allow explicit derivatives. Thus, maximizing the log-likelihood with respect to $\left(\alpha, \beta_{3}\right)$ and the smoothing parameter $v$ using Newton-Raphson approach with analytical derivatives is proved to be a difficult task. We use the Davidon-Fletcher-Powell (DFP) method, which only requires evaluations of the objective function to search for an optimum point. This is a quasi-Newton method that builds up an approximation of the inverse Hessian and is often regarded as the most sophisticated approach for solving unconstrained problems. Earliest schemes for constructing the inverse Hessian was originally proposed by Davidon (1959) and later developed by Fletcher and Powell (1963). It has the interesting property that, for a quadratic objective, it simultaneously generates the directions of the conjugate gradient method while constructing the inverse Hessian. The method is also referred to as the variable metric method. The DFP method has theoretical properties that guarantee superlinear (fast) convergence rate and global convergence under certain conditions. It should be noted however, that this method could fail for general non-linear problems. Specifically, DFP is highly sensitive to inaccuracies in line searches and can get stuck on a saddlepoint or may obtain only a local maximum. To address this issue, we used different starting values to examine whether the algorithm converges to the same point.

Finally, all the computations are performed assuming the bivariate probit model, but similar calculations can be done for the bivariate logistics model.

\section{References}

Albert, P.S., Follmann, D.A.: Modeling repeated count data subject to informative dropout. Biometrics 56, 667-677 (2000)

Ashford, J.R., Sowden, R.R.: Multivariate probit analysis. Biometrics 26, 535-546 (1970)

Brent, R.P.: Algorithms for minimization without derivatives. Prentice Hall, Englewood Cliffs, NJ (1972)

Burton, S.W.: A review of the Fluvoxamine and its uses in depression. Int. Clin. Psychopharmacol. 6(Supplement 3), 1-17 (1991)

Catalano, P.J., Ryan, L.M.: Bivariate latent variable models for clustered discrete and continuous outcomes. J. Am. Stat. Assoc. 87, 651-658 (1992)

Cook, R.: Assessment of local influence. J. Roy. Stat. Soc. B 48, 133-169 (1986)

E) Springer 
Dale, J.: Global cross-ratio models for bivariate, discrete, ordered responses. Biometrics 42, 907-917 (1986)

Davidian, M., Giltinan, D.M.: Nonlinear models for repeated measurement data. Chapman \& Hall Ltd (1998)

Davidon, W.: Variable metric methods for minimization. Technical Report ANL-5990, Argonne National Laboratory, Argonne, IL (1959)

Diggle, P., Kenward, M.G.: Informative dropout in longitudinal data analysis (with discussion). Appl. Stat. 43, 49-93 (1994)

Efron, B., Tibshirani, R.: An introduction to the bootstrap. Chapman and Hall, London (1993)

Ekholm, A., Jokinen, J., McDonald, J.W., Smith, P.: Joint regression and association modelling of longitudinal ordinal data. Biometrics 59, 795-803 (2003)

Fletcher, R., Powell, M.J.D.: A rapidly convergent descent method for minimization. Comput. J. CJ-6(2), 163-168 (1963)

Kenward, M.G., Goetghebeur, E.J.T., Molenberghs, G.: Sensitivity for incomplete categorical data. Stat. Model. 1, 31-48 (2001)

Lesaffre, E., Molenberghs, G., Dewulf, L.: Effects of dropouts in a longitudinal study: an application of a repeated ordinal model. Stat. Med. 15, 1123-1141 (1996)

Lesaffre, E., Todem, D., Verbeke, G.: Flexible modelling of the covariance matrix in a linear random effects model. Biometrical J. 42, 807-822 (2000)

Lindsay, B.G.: The geometry of mixture likelihoods: A general theory. The Annals of Statistics 11, 86-94 (1983)

Little, R., Rubin, D.: Statistical analysis with missing data. John Wiley and Sons, New York (1987)

Little, R.J., Rubin, D.B.: Adjusting for nonignorable drop-out using semiparametric nonresponse models: comment. J. Am. Stat. Assoc. 94, 1130-1132 (1999)

McCullagh, P., Tibshirani, R.: A simple method for the adjustment of profile likelihoods. J. Roy. Stat. Soc. B 52, 325-344 (1990)

Molenberghs, G., Kenward, M.G., Lesaffre, E.: The analysis of longitudinal ordinal data with nonrandom dropout. Biometrika 84, 33-44 (1997)

Morrell, C., Pearson, J.D., Brant, L.J.: Linear transformations of linear mixed effects models. Am. Stat. 51, 338-343 (1997)

Newton, M., Quintana, F., Zhang, Y.: Nonparametric Bayes methods using predictive updating. Springer, New York, (1998) in practical nonparametric and semiparametric bayesian statistics, dey, d., müller, p., and sinha, d.- edition.

Newton, M., Zhang, Y.: A recursive algorithm for nonparametric analysis with missing data. Biometrika 86, 15-26 (1999)

Plackett, R.L.: A class of bivariate distribution. J. Am. Stat. Assoc. 60, 516-522 (1965)

Rotnitzky, A., Scharfstein, D., Su, T.-L., Robins, J.: Methods for conducting sensitivity analysis of trials with potentially nonignorable competing causes of censoring. Biometrics 57, 103-113 (2001)

Scharfstein, D.O., Rotnitzky, A., Robins, J.M.: Adjusting for nonignorable drop-out using semiparametric nonresponse models (c/r: P1121-1146). J. Am. Stat. Assoc. 94, 1096-1120 (1999)

Tao, H., Palta, M., Yandell, B., Newton, M.: An estimation method for the semiparametric mixed effects model. Biometrics 55, 102-110 (1999)

Ten Have, T., Morabia, A.: Mixed effects models with bivariate and univariate association parameters for longitudinal bivariate binary response data. Biometrics 55, 85-93 (1999)

Ten Have, T.R., Reboussin, B.A., Miller, M.E., Kunselman, A.: Mixed effects logistic regression models for multiple longitudinal binary functional limitation responses with informative dropout and confounding by baseline outcomes. Biometrics 58, 137-144 (2002)

Todem, D., Kim, K., Lesaffre, E.: Latent-variable models for longitudinal data with bivariate ordinal outcomes. Technical Report 169, Department of Biostatistics and Medical Informatics, University of Wisconsin-Madison (2002)

Verbeke, G., Molenberghs, G., Thijs, H., Lesaffre, E., Kenward, M.: Sensitivity analysis for nonrandom dropout: a local influence approach. Biometrics 57, 7-14 (2001)

Wu, M.C., Carroll, R.J.: Estimation and comparison of changes in the presence of informative right censoring by modeling the censoring process (corr: V45 p1347; V47 p357). Biometrics 44, 175$188(1988)$ 